\title{
The Brazilian Virtual Observatory - A New Paradigm for Astronomy
}

\author{
R.R. de Carvalho ${ }^{1}$, R.R. Gal ${ }^{2}$, H.F. de Campos Velho ${ }^{1}$, H.V. Capelato ${ }^{1}$, F. La Barbera ${ }^{3}$, \\ E.C. Vasconcellos ${ }^{1}$, R.S.R. Ruiz ${ }^{1}$, J.L. Kohl-Moreira ${ }^{4}$, P.A.A. Lopes ${ }^{5}$ and M. Soares-Santos ${ }^{6}$ \\ Manuscript received on September 09, 2009 / accepted on January 20, 2010
}

\begin{abstract}
We present an overview of current and future Brazilian contributions to an emerging paradigm in astronomy, the Virtual Observatory (V0). Astronomy will soon accumulate an unprecedented amount of data, on the order of $100 \mathrm{~PB}$, while adding 2-4 PB/year - an astonishing five orders of magnitude greater than in 2000. The VO is a response to the astronomical community's demands for improved and homogenized access to these data, combined with the tools to manipulate and explore them. It is a complex enterprise with a decentralized, webcentric nature, implying that astronomers need to rethink the old ways of conducting their scientific programs. Today an international effort is coordinated by the International Virtual Observatory Alliance (IVOA). In Brazil, the National Institute for Science \& Technology (INCT-Astrophysics) recently created by the Ministry of Science \& Technology (MCT) is taking the lead in developing BRAVO (The BRAzilian Virtual Observatory). At the National Institute for Space Research (INPE), we are concentrating our efforts on three distinct aspects of $\mathrm{V} 0$ development: database development and basic infrastructure, data grid and processing grid implementation, and data mining. This paper describes our approach to creating a roadmap for the $\mathrm{V} O \mathrm{in}$ Brazil and some technical developments on which we have already embarked.
\end{abstract}

Keywords: Virtual Observatories, Machine Learning Algorithms, Network Infrastructure, Data Grid, Data Processing, Data Analysis.

\footnotetext{
${ }^{1}$ Instituto Nacional de Pesquisas Espaciais (INPE/DAS), Brazil.

${ }^{2}$ Institute for Astronomy, University of Hawaii, USA.

${ }^{3}$ Osservatorio Astronomico di Capodimonte, Italy.

${ }^{4}$ Observatório Nacional/MCT, Coordenação de Astronomia, Brazil.

5 Observatório do Valongo, Universidade Federal do Rio de Janeiro, Brazil.

${ }^{6}$ Instituto de Astronomia, Geofísica e Ciências Atmosféricas, Universidade de São Paulo, Brazil.

E-mails: rrdecarvalho2008@gmail.com, rgal@ifa.hawaii.edu, harold0@lac.inpe.br, hugo@das.inpe.br, flabarber@gmail.com, eduardo.vasconcellos@lac.inpe.br, renata@lac.inpe.br, j|kohlm@gmail.com, paal05@gmail.com, marcelle.soares.santos@gmail.com
} 


\section{INTRODUCTION}

Astronomy is now an enormously data-rich science, and currently produces terabytes of raw data per day, with a few petabytes already in various archives. Both the data volume and data rate are increasing exponentially, with a doubling time of 1.5 years. Even more important is the growth of data complexity (expressed, e.g., as the dimensionality of the parameter space spanned by the measurements of the detected sources) and heterogeneity. These data are now being federated in a global data grid under the umbrella of the Virtual Observatory (VO). A complete and effective scientific exploitation and exploration of these large and complex data spaces is a highly non-trivial task, requiring a new generation of software (databases, scalable data mining tools, interfaces), hardware (computing power, storage, network infrastructure), and expertise. The absence of these resources is a key bottleneck in data-rich astronomy: the data are there, but the means of extracting knowledge from them are not.

Figure 1 demonstrates the severity of these problems. We see the rapid increase in data volume from only a decade ago, where the Digitized Second Palomar Observatory Sky Survey provided single-epoch observations of half the sky in just 3 bands, to current projects like Pan-STaRRS1, which provides imaging of three-quarters of the sky in 5 filters but now at hundreds of epochs. Including the time domain not only increases the storage and computational requirements, but challenges the community with the need for new algorithms and tools. Incredibly, we see that astronomy is generating data at the same pace as experiments in particle physics. This is extraordinary, considering that the number of researchers and the worldwide financial investment is much less in astronomy. Figure 1 clearly exhibits the necessity of efficient data storage, data processing and data mining, which are specific areas addressed by this project.

Typical research paths taken in the scientific exploitation of large sky surveys are either construction of statistical samples of objects or populations of interest (e.g., normal galaxies, quasars, etc.) and their study (e.g., to probe their evolution, large-scale structure, etc.), or selection of interesting targets (e.g., peculiar galaxies, distant quasars, brown dwarfs, supernovae, etc.) for follow-up observations. The scientific potential of such studies is greatly enhanced by federating data sets (e.g., combining optical, infrared, and radio sky surveys), which often reveal important features and populations of objects not easily distinguishable in any of the data sets taken separately. For example, a typical V0 data enabled project would be a complete clustering and correlation analysis of combined source catalogs, using a federation of multi-wavelength data from several major astronomical surveys, ranging from radio, through infrared, optical, UV, to X-ray, or even $\gamma$-ray. Data federation of the source catalogs from these surveys generally results in a parameter space of $10^{8}-10^{9}$ data vectors in $\sim 10^{2}-10^{3}$ dimensions. The existing tools and algorithms do not scale well to such hyper-dimensional data sets, so we must assemble, test, improve, and deploy the necessary data mining, statistical, and visualization tools for this exploration. Concurrently, we must develop the necessary computational and network infrastructure and human expertise to develop, implement, and utilize these tools. Examples of specific challenges will be presented later in this paper.

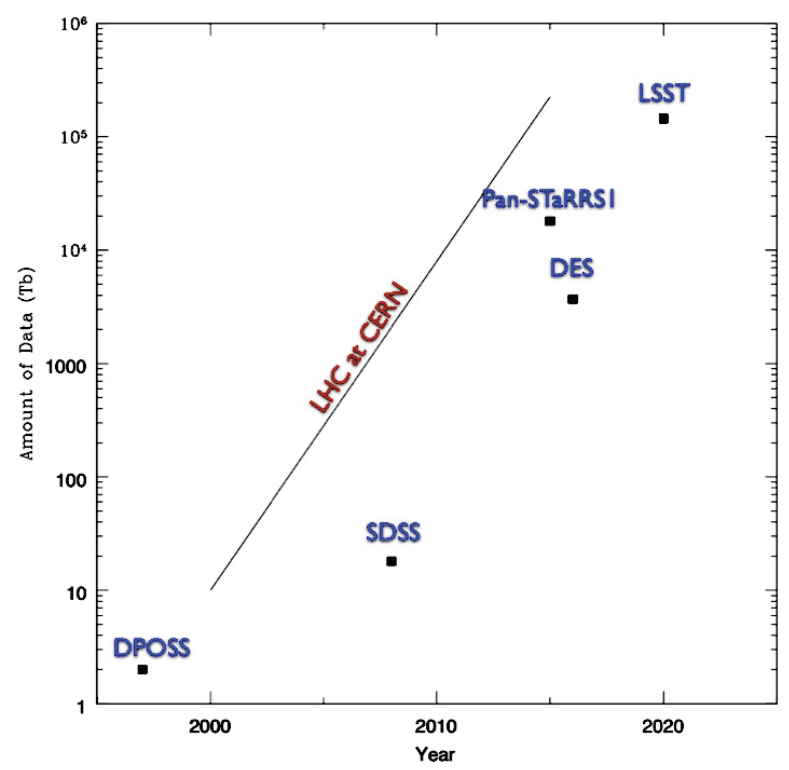

Figure 1 - The rapid increase of astronomical data, considering only the most important optical surveys carried out in the past 20 years. For comparison we show the data rate from the Large Hadron Collider experiment at CERN (for details see http://lhc.web.cern.ch//hc/).

The main objective of the BRAVO@INPE project is to address these strategic issues. More specifically, this project intends to generate investment in information technology, with particular emphasis on Computational Infrastructure, Data Grid, Data Processing, and Data Mining. We present not only a brief history of what has been done in the recent past but also elucidate the specific needs for the near future. This effort aims to prepare the Brazilian astronomical community for the avalanche of data and massive data processing needs that are a reality now, and which will increase rapidly in the coming years with the advent of the large telescopes and surveys currently under development (GMT, TMT; LSST, Pan-STaRRS, VISTA, VST).

This paper is organized as follows: section 2 outlines the 
general concept of the Virtual Observatory, establishing a context for the more specific components described later. Section 3 describes the initial stages taken in generating a roadmap for the VO in Brazil, while section 4 introduces two of the basic elements of any V0: computational infrastructure and databases. The fundamental concepts of data grids and processing grids are presented in section 5. Section 6 describes an image processing pipeline developed by our group, 2DPHOT, and the main characteristics of the Astro-Wise environment. Section 7 provides an overview of the ongoing developments within Brazil in terms of astrophysical applications. Section 8 reviews data mining and describes specific projects we are undertaking in this field. Section 9 focuses on the four main areas in which we plan to invest resources, while section 10 summarizes the BRAV0@INPE project.

\section{THE VO CONCEPT}

For more than two decades, the international astronomical community has witnessed an exponentially growing capacity for accumulating astronomical data. Today, information is gathered in large surveys from the ground and from space, covering virtually the entire electromagnetic spectrum, from X-rays through the ultraviolet, optical, infrared, and beyond. Individual projects yield complementary data through specific, targeted scientific programs. Much of these data are made available to the community through public servers, usually in several different formats, and distributed at many institutions. The data quality, metadata, interfaces, and accessibility are heterogeneous, since each project typically curates its own data, presents it in a custom database, and even data formats in astronomy are instrument dependent with little effort made to unify them.

An underlying concept of the $\mathrm{V} 0$ is that by providing improved and homogenized data access combined with the tools to manipulate and explore the data, the need for new observations will be reduced even as the scientific output is increased. All gathered data can be accessed via the VO, enriching the international community. Large surveys would take precedence over individual, targeted observations, providing added coherence to the VO structure. Therefore, the $\mathrm{V} O$ is not an enterprise driven by a single institute or even one country. It is rather a community endeavor aimed at the democratization of information that will certainly expand to other scientific areas like meteorology, geophysics and space science, allowing new interactions and the exchange of methods and technology. Thus, the V0 today represents to the astronomical community what the Internet was for the academic world in the 1980s. It is clear today that science, especially in developing countries, would be shockingly different without the Internet in the same way that we envisage in the future saying that astronomy would not be the same without the VO.

The Virtual Observatory (VO) concept is the astronomical community's response to the scientific and technological challenges posed by massive and complex data sets. At its heart, the VO is a set of standards - metadata (data describing data), interoperability, and other minimum requirements to be a V0-compliant database [11]. Such standards allow the development of VO tools that can then be deployed to operate on any VO compliant data set. Disparate surveys and individual observers' programs can thus be federated, queried, and manipulated by a single tool. Achieving these basic goals is not simple. To exemplify the obstacles to dealing with a modestly large amount of data, its complexity, and the challenge of processing it over a reasonable timescale, we examined the re-processing of reduced galaxy images from the seventh and final data release from the Sloan Digital Sky Survey (SDSS DR7). These data cover $\sim 8400$ square degrees on the sky and provide images in five bands. We consider only the re-measurement of photometric parameters using a custom pipeline (described later) - not the reprocessing of raw data to calibrated images, and ignore the spectroscopic data entirely. Even for this modest task, if we want to process the imaging data in only one band, in one week, we would need 6577 processors, which is a factor of 15 more computing power than everything currently available to Brazilian astronomers. We use a timescale of one week as an upper limit for what a user would accept to retrieve important information from such a large data set - and this data set is almost trivial compared to upcoming surveys.

Computational hardware requirements are just one small part of the issues that arise when dealing with such vast data sets. Processing takes a lot of time, so once completed, it is of paramount importance that querying and retrieving data be done quickly. This requires investment not only in database software, but the astronomical and computational expertise to design and implement efficient and scientifically useful data models. This information, once structured in such a database, needs to be retrieved efficiently, demanding high-speed internet connections to which most research centers in Brazil do not have access. For these reasons, our top priorities include implementing grid computing to enable the processing of massive data sets; creating a dedicated network for astronomy to enable access to the resulting data, and training astronomers and computer scientists to develop these tools to produce cutting-edge science. Figure 2 summarizes this critical situation. 


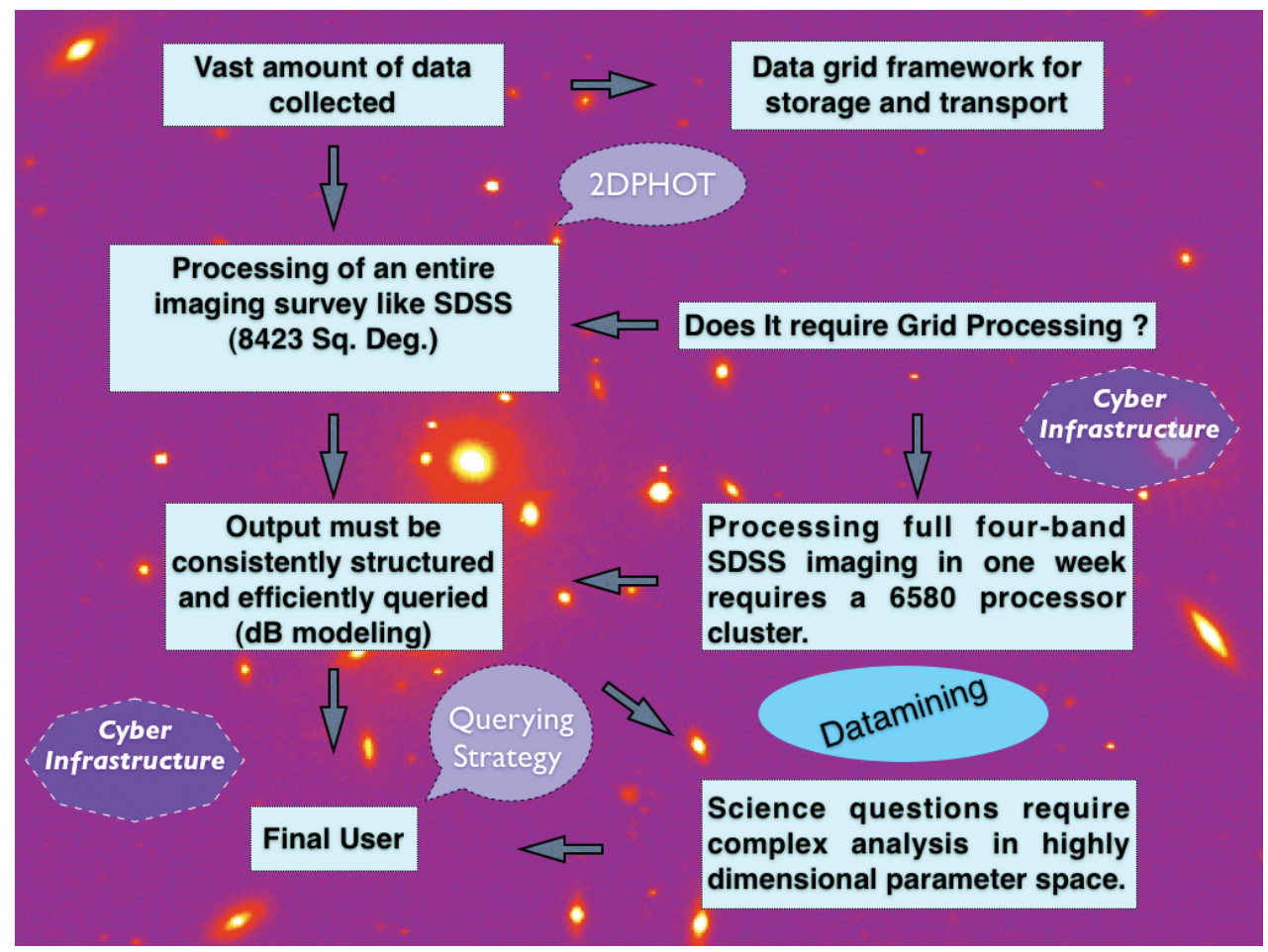

Figure 2 - The complexity of implementing a V0 structure considering all the infrastructural elements and addressing the demands of the astronomical community. The needs expand rapidly and will grow beyond the computational resources currently available, especially in Brazil.

Nevertheless, many V0 compliant databases and tools have already been developed. DS9, a commonly used astronomical image viewer, can communicate with databases using V0 protocols. The European Southern Observatory has developed VirGO, a visual tool that allows both amateur and professional astronomers to search for available data in any part of the sky using planetarium-style software, and then retrieve data based on user supplied constraints. The US Virtual Observatory provides tools to convert ASCII tables to VOTable format, search multiple databases around a given position with one query, cross-match objects across databases, and more. One thing is clear - while the VO today provides standards and tools to find and extract data, few VO compliant tools exist to analyze the data. As we describe, the Brazilian contribution to the VO will focus on deployment of such resources.

\section{THE STATUS OF INFORMATION AND COMMUNICA- TION TECHNOLOGIES (ICT) IN BRAZIL}

The new era of large data sets and the co-requisite data processing needs led to the recognition two years ago that we must modernize the tools for astrophysics in Brazil. In addition to the large photometric and spectroscopic surveys being carried out in both hemispheres, Brazil has committed significant resources to new facilities (including SOAR, Gemini, BDA, etc). As a result, we have access to extraordinary amounts of data in all portions of the electromagnetic spectrum, but without standard techniques for storage, retrieval, distribution, processing or analysis. Thus, the underlying concept of BRAV0@INPE is to federate these resources, using a common framework, standard interfaces, computational infrastructure and analysis tools. BRAV0@INPE is a branch of BRAVO, which is part of INCT. BRAVO comprises a committee in charge of planning VO activity in Brazil, with branches located at various universities and institutes (e.g. BRAV0@IAG at Univ. of São Paulo, BRAV0@0N at Observatório Nacional). BRAV0 orchestrates and coordinates the specific developments at different branches.

All of these developments are embraced within the concept of Information and Communication Technologies, encompassing all means for processing and communicating information. ICT is often used to describe digital technologies including methods for communication, transmission techniques, communications equipment, and techniques for storing and processing information. The term has gained popularity partially due to the convergence of information technology (IT) and telecom technology. 
Before embarking on a major enterprise to develop BRAV0@INPE, we must understand our current hardware/software/personnel resources and their ability to meet our needs both today and in the future. The partners in this project are the thirty-one institutes comprising the INCT-Astronomy (National Institute for Science \& Technology) recently created by the MCT (Ministry of Science \& Technology). The central repository of knowledge about computational hardware, software and personnel in BRAV0@INPE will be the BNPGA (Brazilian Network for Processing Grid in Astronomy). Appendix A lists the institutes that will compose the BNPGA and their representatives. This new program will allow us to trace the roadmap of what is really needed for the future.

We have performed an initial census of the capabilities of the INCT-Astronomy member institutes. Here we provide a brief synopsis of the results; the complete list of questions and results can be found in Appendix B. We find that most users of our community have access to at least a desktop computer with moderate computational capacity. This conclusion must be seen with caution, as many members of our community use data of low complexity and in small volumes. This situation is changing dramatically with the next generation of large surveys and telescopes. In this context, the current computational facilities may be adequate today, but it is clear that the current cyber-infrastructure will be obsolete when dealing with the extremely large amount of data coming from both stellar and extragalactic projects.

These new programs will often require large computing clusters. We examined access to modern servers with more than 8 processors each (Class $A$ ) and to beowulf types, composed of mono-processed nodes and internal networks of $100 \mathrm{Mbps}$ (Class B). Only 12 out of the 20 institutes that responded have access to a cluster and only 7 out of these 12 have access to a Class A cluster. It is important to note that in some cases the clusters are shared with researchers from different disciplines like Physics since the small groups of researchers developing Astronomy in Brazil are contained within large Physics departments.

Adding up all the available processors in the different clusters gives, in principle, the total number of processors available for grid processing (see Appendix B, Table 4). This total, 419, is only $6 \%$ of the required number to processing the entire SDSS DR7 in one band, in one week, for example. This is only a crude estimate considering that all the processors are different, some better than others - fifty are old types of processors that would add little to the total processing capacity. It is to be noted that modern surveys like Pan-STaRRS use a single 512-node cluster with the latest $3+\mathrm{GHz}$ processors to analyze their data - more powerful than all of the
Brazilian astronomical community's computers combined.

Beyond processing power, the total disk storage available to our clusters is approximately 45TB. While this satisfies the needs of individual groups, it is clearly incompatible with the needs of the coming decade where telescopes will produce data at a rate of $2 \mathrm{~PB} /$ year. Moving any fraction of such data quantities also requires high-speed network connections, which many of our institutions still do not have.

The results of this census demonstrate the extreme deficiency of the current hardware, software and network infrastructure in Brazil. An often overlooked (and underfunded) aspect of any computational project is the need for personnel with expertise in all aspects of the program. In BRAV0@INPE, we cannot expect a computer scientist with experience in commercial database applications to understand and implement astronomical databases without new training. Similarly, we would not expect an astronomer to develop efficient computational algorithms for, say, clustering analysis, without learning about recent advances in such applications.

To address some of these issues, we organized two workshops in 2007 at INPE where formal presentations were given by a number of researchers from around the world engaged in escience. At the second workshop we had specific presentations by researchers from Brazil engaged in V0-related projects, showing the tremendous potential that we have to actively participate in this international effort (see www.ivoa.net). For more information and access to the presentations of the lectures see www.lac.inpe.br/projetos/bravo/. A major component of this project will be the training of technical staff, which is of paramount importance for us. We have already begun a program of visits by Brazilian astronomers and computer scientists to foreign institutions with extensive astronomy database involvement, including Caltech, Johns Hopkins University, and the Institute for Astronomy in Hawaii. The recent inclusion of BRAVO within the IVOA will help increase the interaction with other foreign groups.

\section{DATABASE DEVELOPMENT AND BASIC INFRASTRUCTURE}

Many scientists view databases as simply a form of data storage. Perhaps you could do simple computations on columns on your personal computer and output the results. Typical tables might have tens to thousands of entries. Even a large food market has only about 50,000 different items available - and we are tempted to imagine that their warehouse database must be large and complex. We would be very wrong. 
Astronomical data sets have far surpassed the largest commercial databases in size and complexity. Almost twenty years ago the Digitized Second Palomar Observatory Sky Survey [6] database contained over 100 million objects, measured in three bands, with a total of 100 properties per object. Information about the survey (calibration, plate metadata, related CCD imaging, classification schemes) was spread over $\sim 50$ different tables. Information from these tables often had to be joined (such as calibration and raw fluxes) to yield scientifically meaningful data. This database never became easily accessible to the public, which would have required the creation of an added layer of interfaces and query tools.

The current gold standard of databases in astronomy is the Sloan Digital Sky Survey. The imaging catalog has almost half a billion objects, in five filters, with nearly 500 columns of data on each object. While the volume of this single table (many TB) is itself daunting, the SDSS database has nearly 100 unique tables, with an additional 50 views offering easy access to scientifically useful subsets of specific tables. The complexity of this database required years of consideration to design a workable schema, decide on which columns to generate indices to speed up queries, understand how to load and update tables with new data, and how to provide public access. Just writing a portion of the table documentation was a full time job for a postdoctoral researcher for almost two years. Beyond the nearly 20TB of catalog data, SDSS also allows users to access a comparable volume of images.

While the SDSS is quite complicated for an astronomical database, it pales in comparison to those from next-generation projects. Upcoming surveys such as Pan-STaRRS and LSST will yield a comparable amount of data - every time they survey the sky. These projects will create a new SDSS every few months. Not only do they produce multi-filter imaging, which must be processed, cataloged, stored, and distributed, they will also produce time series data. Every object detected in one image must be matched to its corresponding detection in all earlier images of that same area. Optimal methods for differencing images must be developed to look for astronomical sources that vary or move. An entire pipeline is necessary to take moving objects, find them at different locations in images taken at different times, associate them, and generate orbits. Light curves for both stationary and moving objects must be created. All of this must be done almost instantaneously, because rare, one-time events such as supernovae must be found and notifications for follow-up observations disseminated before they fade. This means processing one gigapixel image every minute. The resulting database is correspon- dingly more difficult to model and populate. A static sky database must be created with everything detected, and updated as repeated observations allow for the creation of ever deeper images. Variable and moving objects must have all of their detections stored so that light curves and orbits can be derived.

The evolution of these surveys vividly demonstrates that we must contend with a new paradigm in astronomy. We must have the resources to store, disseminate and access large databases. We must have the knowledge of how such databases are structured, and how we can develop our own tools to create novel science. We must have our own databases for Brazilian programs, and enable interoperability with VO tools to maximize their scientific potential. We must also remember that so far we have only discussed large optical surveys. Multi-wavelength and multi-epoch studies demand new tools to cross-identify sources observed across the electromagnetic spectrum, with different spatial and time resolutions. This fundamental problem too has been approached but is far from solved. In all of these arenas, Brazil has much to learn, but also much to contribute.

\section{DATA GRID \& PROCESSING GRID}

We are in the midst of a revolution in data gathering that encompasses all realms of science. In particular, the volume of data in astronomy, both real and simulated, is growing exponentially. The need for tools to analyze these data is naturally creating a new branch of scientific investigation - data science. There are many challenges in this emerging enterprise. We must have methods for extracting knowledge from large amounts of data, which by itself is non-trivial. What is important and what is noise? Which correlations are fundamental and which are secondary? In addition, we must urgently develop the skills and tools for processing these data. These requirements are already being addressed by two areas of computer science: data mining and high performance computing (HPC). We discuss the former in Section 8; here we will focus on the latter.

There are many approaches to HPC. The first one used parallel machines - vector machines, multi-processing machines with shared memory, multi-processing machines with distributed memory, and more recently multi-core processing chips. These solutions aimed to improve the processing capacity of a single, central machine. By the late 1990s, a form of distributed computing was created, using internet connections among geographically distributed processors to spread the computational labor. This is the underlying concept of grid computing, where processors across a city, country, or the whole world can be utilized by a single pro- 
gram. This type of grid is a new environment for science in the current century. We should note that a computing grid is not properly a HPC implementation. This concept (HPC) is typically reserved for enhancing the performance of a single system. However, grid computing is one of the strategies for addressing the need for intensive computation.

There are many types of grids and generally they can be classified according to:

- the nature of the processing: data grid or processing grid;

- he focus of the processing: open vs. closed or general vs. dedicated;

- the hardware components: homogeneous or heterogeneous.

Since the accumulation rate of data in astronomy is already reaching an unprecedented level of $10 \mathrm{~PB} / \mathrm{year}$, it is becoming difficult, technically and financially, to centrally store all of the data, and impossible to replicate data for personal use. A data grid provides an environment for distributing, sharing, and modifying large amounts of data. We find applications for such a grid in different fields, such as meteorology. Examples include the Earth System Grid (http://www.earthsystemgrid.org/) and SegGrid (http://seghidro.Isd.ufcg.edu.br/). In astronomy, the Montage software platform (http://montage.ipac.caltech.edu) has been prepared to run in a grid environment. Many other astronomical tools have been or are being ported to grid applications [2, 36].

Similarly, increasingly large and complex processing tasks are required to process and analyze these data sets, or to generate large simulations. A processing grid addresses these issues. Collaborative processing is a type of application that exemplifies this new technology. The SETI@home project (Search for Extra-Terrestrial Intelligence, http://setiathome.ssl.berkeley.edu/) was the first popular distributed computing project, and now has over 3 million users, hosted by the Space Sciences Laboratory (University of California, USA). It is only one of 50 such projects using the BOINC volunteer and grid computing platform (http://boinc.berkeley.edu/). The Large Hadron Collider (LHC) has developed an enormous computing grid joining 170 computing centers in 24 countries (http://lcg.web.cern.ch/lcg/). Processing grids in other fields include protein folding (http://folding.stanford.edu/), climate change

(http://www.climateprediction.net/), and seasonal mesoscale climate prediction GBRAMS

(http://www.cptec.inpe.br/brams/gbrams.shtml) and RECLIRS (http://yule.lacesm.ufsm.br/nucleus332/). Numerous online directories of grid computing projects

(http://www.gridcomputing.com, for instance) provide lists of dozens to hundreds of grid projects (commercial and scientific), environments, and applications.

Thus, it is imperative that we take advantage of the computing resources available at different computer centers linked by fast network connections. This could take the form of our own, internally developed grid implementation, or the installation and deployment of existing tools such as BOINC. Today, Brazilian users contribute almost 10 Teraflops of computing power to BOINC projects, the highest in South America. One of the main goals of BRAV0@INPE is to create a processing grid to harness academic computing along with this private processing power, with initial focus on two specific astrophysical applications: image processing with 2DPHOT (described in Section 6.1); and analysis of cosmological simulations with hundreds of millions of particles using the FoF algorithm (described in Section 7.2).

Our team is currently strongly committed to the use of grid technologies and web services within the V0 context. Specifically, our focus is data modeling, within the scope of BRAV0@INPE, to develop a framework for the metadata describing both observed and simulated data. We examine the logical relationships between these metadata, with the intent of establishing a general architecture for retrieving, processing, and interpreting data from different branches of spatial science and in particular from astronomy. This is an important step for constructing protocols that will guide vo applications.

\section{DATA PROCESSING}

As described above, critical issues in V0 development include the large amount of data and how it is to be processed - transformed from raw images to reduced data suitable for further analysis. Here, we describe two concrete steps to address these problems undertaken in Brazil: the installation and operation of the first Astro-Wise (AW) node in South America and the insertion of our photometry environment (2DPHOT) into AW.

\subsection{DPHOT}

2DPHOT is an automated tool to derive both integrated and surface photometry of galaxies in an image, to perform reliable star/galaxy separation with accurate estimates of contamination at faint fluxes, and to estimate the completeness of the resulting catalog. A 2DPHOT graphical user interface (2DGUI) is also under development, allowing the user to easily set 2DPHOT input opti- 


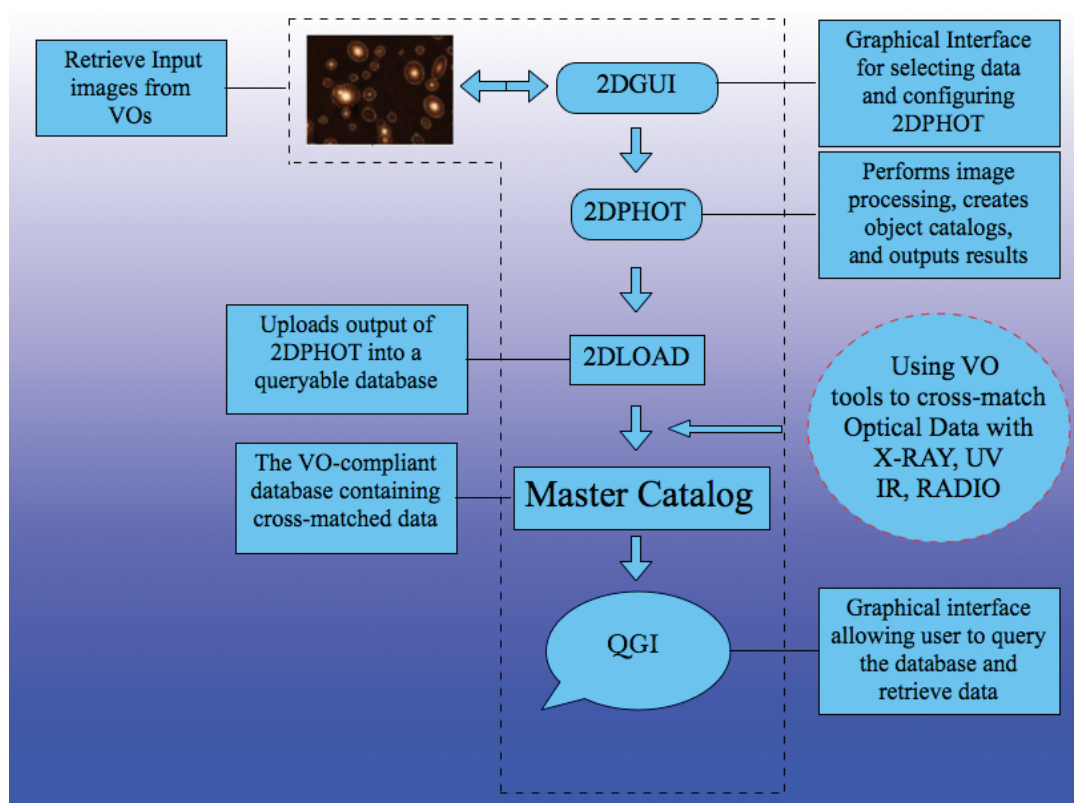

Figure 3 - Schematic representation of the 2DPHOT environment.

ons and detection parameters. More details can be found in [20]. We show a schematic representation of the 2DPHOT environment in Figure 3.

The main tasks of 2DPHOT are:

- Producing a cleaned catalog of the image.

- Performing reliable star/galaxy classification.

- Estimating the completeness of the galaxy catalog and the contamination due to star/galaxy misclassification.

- Constructing an accurate model of the Point Spread Function (PSF) of the input image, taking into account possible spatial variations of the PSF as well as deviations of stellar isophotes from circularity.

- Deriving structural parameters of galaxies by fitting galaxy images with two-dimensional PSF-convolved Sérsic models.

- Measuring galaxy isophotes by fitting them with Fourierexpanded ellipses, and deriving one-dimensional surface brightness profiles of galaxies.

- Measuring the growth curve of seeing corrected aperture magnitudes for galaxies.

The image analysis flow of 2DPHOT is presented in Figure 4. 2DPHOT is being utilized by several projects conducted by researchers within and outside of BRAVO. In a spectroscopic and photometric study of a rich cluster at intermediate redshift, it is used to measure global properties of cluster galaxies [26]; it is also used in a fundamental plane study based on SDSS and UKIDSS data [21]. The analysis of internal color gradients in early-type systems has been recently published in [23]. We also used 2DPHOT in a recent study of Fossil Groups [22]. We have also begun a large-scale study (SPIDER; Spheroids Panchromatic Investigation in Different Environment Regime) of the general properties of early-type galaxies (ETGS) combining SDSS and UKIDSS data. This project makes extensive use of 2DPHOT to properly measure the seeing corrected structural parameters for nearly 40,000 ETGs.

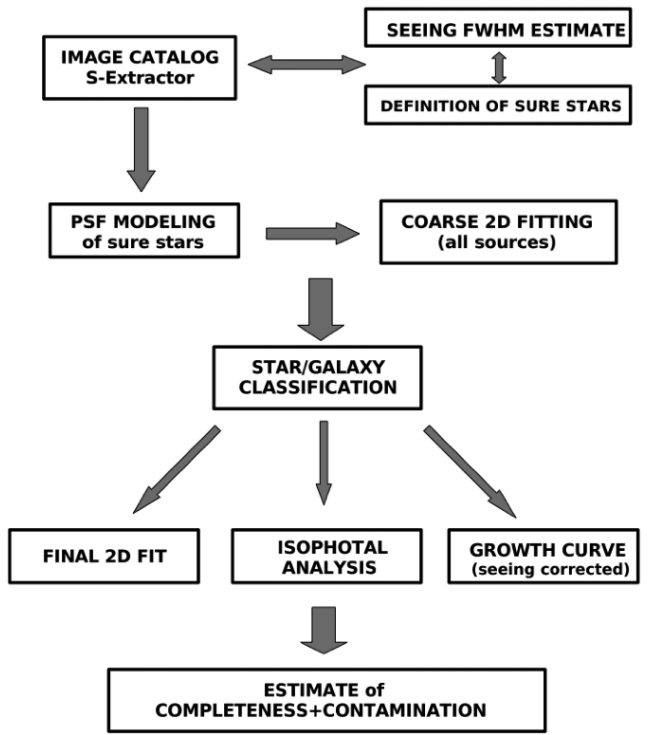

Figure 4 - Image Analysis flow of 2DPHOT. 


\subsection{Astro-Wise}

2DPHOT is only a starting point in preparation for the avalanche of data in the next few decades. 2DPHOT requires as input an already processed image. Thus, we must be able to process raw images either on an individual basis or in a pipeline. To do so, we are taking advantage of the Astro-Wise (AW) system, developed by a consortium of European astronomy research institutes. The AW environment consists of hardware and software federated over five institutes in Europe, designed to scientifically exploit the increasing amount of data produced by experiments in different fields. AW is a general information system which was initially geared towards astronomy, but is now also used in other branches of science. This is an essential trait of AW in the context of a unified environment for data processing at INPE. It allows a user to archive raw data, calibrate data, and perform post-calibration scientific analysis. All results are stored in a single environment that links together all of the discrete steps performed when analyzing a data set. This complete linkage, including the input, output, and software code used to derive one from the other, for arbitrary data volumes, has only been feasible thanks to a novel paradigm devised by the creators of AW. The algorithms included in the software have been developed to include arbitrary optical wide field imagers. This aspect is of major importance for BRAV0@INPE, since we will be developing software that enables us to ingest data from instruments available at SOAR and in the future from LSST.

AW was designed and implemented as a fully scalable and distributed information system to properly handle the huge amount of data that will be produced by large area surveys in the near future. By allowing the end-user to trace the data products, following all dependencies from the final catalog back to the raw data, it becomes possible to re-derive the result with better calibration and/or improved analysis tools. This represents perhaps the first time that astronomers could truly reproduce each others results.

To achieve these goals, AW includes structural functions that allow for storing data models along with data, in distributed databases [38]. It contains a file server that can access these databases along with the image data, and a processing grid that can utilize parallel clusters while retrieving and storing input and output in the databases. AW is fully scalable so that it can accommodate large and small projects and can work with data from any imaging camera. Adding new analysis code is simple, allowing users to deploy their own tools on the compute clusters accessible to AW. These properties mean that AW overcomes the limitations of traditional analysis tools, which typically reside on a user's own computer or cluster. Reduction processes would usually be run by a single user, and saving sufficient metadata to reproduce every step is up to that individual. These behaviors are simply not sufficient for the new data volumes, collaborations and complexity in modern astronomy. Hundreds of terabytes of data will start entering the system when SOAR starts operating with the complete suite of planned instruments.

\section{DATA ANALYSIS}

The processing of raw data from a telescope into images, spectra or other products suitable for further analysis is only the first computationally intensive step on the path from photons to science. The processed data must be analyzed to detect, classify and characterize individual objects and groups of objects, and obtain physically meaningful measurements.

Within BRAV0@INPE, we are focusing on a few distinct data analysis projects:

- Implementation of a decision tree for star/galaxy separation in the faint magnitude regime for wide field images;

- Development of a parallelized Friends-of-Friends (FoF) algorithm, with application to galaxy catalogs from the SDSS Stripe 82 project (http://www.sdss.org/drsn1/DRSN1_data_release.html)

- Automatic morphological analysis of images in Stripe 82 using both traditional tools for structural parameter estimation (e.g. concentration/ asymmetry, [15]) and advanced methods for image analysis such as the Euler characteristic and gradient spectral analysis [31].

- Development of a cluster finding algorithm using Voronoi Tessellation, but considering a more realistic background distribution instead of the usual Poissonian assumption. Preliminary results were presented in [34].

- Virial analysis of galaxy clusters, allowing us to measure the most important dynamical quantities including total mass, based on the gapper technique described below.

\subsection{Decision Tree (DT)}

A decision tree is a computational method for splitting data into distinct classes, either based on pre-existing knowledge of the subgroups (supervised) or on inherent characteristics (unsupervised). Let a data set be described by a collection of attributes for each object in the data set. Each attribute is a measurement 
of some characteristic of an object (such as magnitude or size). These objects could belong to different classes or clusters (such as stars and galaxies). Imagine a data set for training, where the class of each object is already known. Our task is to develop a classification rule to determine the class of an object based on its various attributes. If two objects have the same attributes, but they belong to different classes, then it is impossible to separate these objects based on this set of attributes. In this case, the data set with these attributes is not appropriate for a training set for the induction task. Thus, we must also determine the appropriate attributes to separate the objects into the desired classes.

Unlike other techniques for clustering analysis, the DT does not rely on distance metrics but instead makes a series of branching decisions based solely on numerical values of the attributes. A DT is a simple structure, where the final leaves define to which cluster an object with a specific set of attributes belongs. The nodes represent tests on a given attribute, with a branch for each possible output. For classifying an object, the starting point is the root of the tree; a test is applied to one attribute and the appropriate output branch is determined. The process is repeated using other attributes until the last leaf. Therefore, the object will belong to the cluster represented by that leaf.

There are many induction algorithms for decision trees. The ID3 algorithm, developed by [28], is the most popular. The algorithm was improved, allowing continuous parameters [29]. A package called WEKA (Waikato Environment for Knowledge Analysis) has been developed where several standard machine learning techniques were incorporated into a "workbench". Several decision trees were designed for classifying objects detected in the SDSS (Sloan Digital Sky Survey) data for 5 passbands (u, g, r, $\mathrm{i}, \mathrm{z}$ ), employing WEKA (see [32, 35];). Our main goal is to provide a $V 0$ service to deal generally with the problem of star-galaxy separation - for any training set provided by the user, allow the generation of an appropriate DT using different methods and cross-validate the final obtained trees.

\subsection{Parallel Friend-of-Friends Algorithms}

The friend-of-friends ( $\mathrm{FoF}$ ) algorithm is commonly used to join galaxies within a linking volume around each galaxy. This method has several attractive features, like being independent of the particular geometry of the galaxy distribution. For a given linking volume a unique group catalog is defined. One of the main problems in using this algorithm is the time it takes to process large numbers of objects, scaling with $\mathrm{N}^{2} \log \mathrm{N}$. It is necessary to weaken this dependence on the total number of objects and thus be able to treat the hundreds of millions of particles found in current large cosmological simulations.

First experiments on reducing the dependence on $\mathrm{N}$ have shown that after a domain decomposition (subdividing the data in redshift shells) combined with a post-processing step we have already reduced the scaling to $\mathrm{N} \log \mathrm{N}^{2}$, a considerable improvement. A simple domain decomposition can be implemented in a purely parallel manner, but it is insufficient because some objects artificially separated by sub-domain boundaries could in reality belong to the same group. Therefore, a post-processing procedure is applied to examine objects close to a boundary but with a valid friend in an adjacent sub-domain. Our parallel version has fully reproduced previous results [5] for computing the potential gravitational energy spectrum for galaxies and clusters of galaxies at many redshifts. A VO service will be made available allowing the user to run the FoF algorithm over the most important cosmological simulations available to date as well as those inputed by the user.

\subsection{Advanced Tools for Morphological Analysis}

As spatial information becomes ever more accessible through high resolution digital images, the need for robust techniques for complex pattern characterization is obvious. An obvious example is the mathematical description of galaxy images. Considerable attention has been paid to morphological classification of E/SO/Sa/Sab/Sm/lrr galaxy morphologies using Sloan Digital Sky Survey imaging. The data to be analyzed usually are (1) skysubtracted, cleaned and log scaled g-band images; (2) filteredenhanced versions of the g-band images; (3) the corresponding RGB composite images; and (4) a set of measured parameters, including surface brightness, position angle, ellipticity and spectral coefficients. In this sense, some useful mathematical and statistical approaches have been proposed (e.g. [25]) to estimate the CAS (concentration, asymmetry and clumpiness) structural parameters. Motivated by the data analysis challenges in the context of BRAV0@INPE, we have developed an alternative and complementary approach for characterization of inhomogeneity and radial asymmetry in galaxy images. Inhomogeneity is calculated using the Euler characteristic from the Minkowski functional. Radial asymmetry is obtained by applying gradient pattern analysis to 2D wavelet multi-resolution samples of the image. The combination of both structural characteristics is proposed as an effective measurement for galaxy morphology. The main objective here is to implement a VO service to deal with morphological analysis in general and in particular to analyze the entire SDSS (DR7) and 
explore the relationships between morphology and stellar population parameters, for instance.

\subsection{A Modified Voronoi Tesselation Code to Search for Clusters of Galaxies}

We are currently developing a cluster finder algorithm in $2+1$ dimensions based on Voronoi tesselation (VT). The method is non-parametric and does not smooth the data, making the detection independent of the cluster shape. It uses all of the available galaxies, going as far down the luminosity function as the input catalog permits. It does not rely on the existence of features such as a unique brightest cluster galaxy or a tight ridgeline in color-magnitude space. It works in shells of redshift, treating each shell as an independent 2-dimensional distribution of galaxies. The core of the VT algorithm is the background above which an overdensity must rise to be identified as a cluster. In contrast to earlier implementations of the VT algorithm, we do not assume a Poissonian background. We use a more realistic assumption that the angular two-point correlation function of the background distribution has a power-law shape, similar to what is actually observed. In a given redshift shell, we build a Voronoi diagram and compare the distribution of cell areas with the distribution expected from a background-dominated eld. We set as a threshold the cell size below which the distribution starts to increase faster than its background counterpart. The clumps of contiguous cells found with density significantly above their respective cells are flagged as potential clusters.

The Voronoi diagram of a 2-dimensional distribution of points is a unique, non-arbitrary and non-parametric fragmentation of the area into polygons. A simple pseudo-algorithm to perform such fragmentation is the following: starting from any point P1, we label its nearest neighbor P2 and follow the perpendicular bisector between those points. We stop when we reach the first point $\mathrm{Q} 1$ on this bisector that is equidistant from P1, P2 and a third data point P3. We now walk along the perpendicular bisector between $\mathrm{P} 1$ and $\mathrm{P} 3$ until we reach the point $\mathrm{Q} 2$ and identify the next point P4 by the same criterion. Successive repetition of this process will eventually bring us back to $Q 1$ after a finite number of steps, creating a polygonal shape, the Voronoi cell, enclosing P1 and having vertices at the points Qi. By repeating this process for each point Pi (every galaxy in the redshift shell) we will have built the VT corresponding to this field. An example is shown in Figure 5, which plots the distribution of galaxies on the sky (black dots) along with the Voronoi cells surrounding each galaxy. There are several robust and efficient computational al- gorithms to build a Voronoi diagram from a given distribution. In our code we use the so-called divide-and-conquer algorithm implemented in the Triangle library [33].

There are no arbitrary parameters in constructing the VT for a given data set. Cells will be smaller in the high-density regions and since each cell contains one and only one point, the inverse of the cell area gives the local density. The VT cluster finder takes advantage of this fact in the process of detection. We plan to implement a VO service where the user can input a galaxy catalog over a given area of the sky and receive a cluster catalog as output.

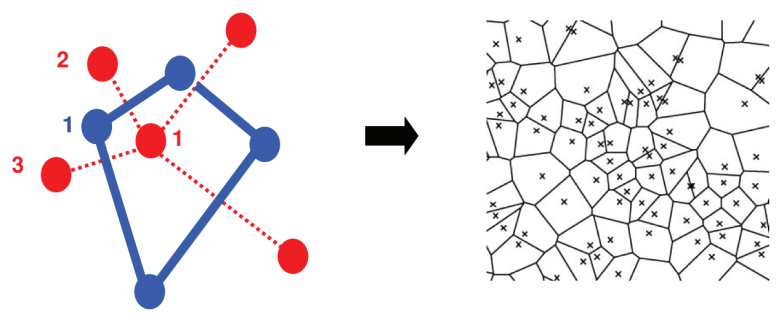

Figure 5 - Diagram showing how Voronoi cells are constructed and a galaxy distribution on the sky with its corresponding tesselation.

\subsection{The Virial Analysis Tool for Understanding Cluster Dynamics}

Removal of interlopers and proper selection of galaxy cluster members is an essential step in the dynamical modeling of clusters and investigations of environmental effects affecting bound galaxies. There are several different approaches for interloper removal available in the literature. A recent comparison of the performance of many different methods applied to N-body cosmological simulations is given by [39]. In particular, they found that differences in mass estimates may be explained by the number of interlopers a given method selects or rejects. These could also explain the discrepant estimates from other methods of mass estimation (e.g., based on X-ray observations or lensing analysis). The shifting gapper method has two main advantages: (i) it is based on combined information for both position and velocity; (ii) it is independent of any hypotheses regarding the dynamical state of the cluster. The procedure we consider is similar to the approach adopted by [8]. The input data consists of the radial and velocity offsets of each galaxy from the cluster center, being visualized as a phase-space diagram. It works through the application of the gap technique $[18,27]$ in radial bins from the cluster center. This technique is used to identify gaps in the redshift distribution, resulting in the identification of groups in z-space. The bin size we consider for the shifting gapper is $0.60 \mathrm{Mpc}$ or larger to force the 
selection of at least 15 galaxies (consistent with [8]). Galaxies not associated with the main body of the cluster are eliminated. This procedure is repeated until the number of cluster members is stable (no more galaxies are rejected as interlopers). After a final list of members is reached, they can be used to measure the cluster velocity dispersion, from which we can estimate the cluster mass through virial analysis. While other procedures are based on physical assumptions about the cluster mass profile, the shifting gapper makes no physical hypotheses about the cluster's dynamical state. Further details of this method can be found in [24]. This technique will be integrated in the VO service described in the previous subsection and will allow the user to carry out a dynamical analysis for the clusters detected with VT and having sufficient redshift measurements.

All of the applications described here already exist or are close to completion. However, they have not yet been deployed as V0-compliant tools. To accomplish this goal we must first implement the foundational concepts of the VO - network infrastructure, grid processing, and most importantly interoperability which is still lacking in the Brazilian astronomical panorama.

\section{DATA MINING}

After the completion of image processing and derivation of meaningful quantities through data analysis, we are now confronted with an enormous collection of numerical quantities describing our data. An extant example is SDSS imaging, with 500 million objects, each with nearly 500 measured attributes. Which of these parameters are connected to fundamental physical properties? How do different types of objects cluster in high-dimensional parameter spaces? How do we find rare classes of objects, especially in the presence of errors or catastrophic mismeasurements? The 21 st century will be a period of data-driven science, with the development of techniques to uncover the hidden knowledge in these kinds of massive data sets. This is the primary concern of a long standing branch of computer science - data mining (DM) - that has been applied extensively to astronomical data. It embraces a set of techniques for dealing with classification (neural networks [3, 13], decision trees [28, 29], clustering analysis [12, 19], visualization [30, 14, 40, 37], pattern recognition $[17,4])$ and statistical analysis of massive data sets with extremely high dimensionality [7, 9]. However, astronomers have yet to implement many of these techniques in easily accessible, cross-database tools. The incredible dimensionality and complexity of astronomical data also challenges conventional implementations of these algorithms [16].
In the space science domain, although there are extensive archival data resources available over the web, the ability of scientists to access and analyze this content is becoming more and more limited. The large data volumes cannot be moved to a personal workstation to be processed by an individual's own software, while the software cannot be easily placed on the data host. Thus, DM in the context of this project refers to specific computational methodologies, working in a logical system, to extract information and find hidden patterns embedded in the large amounts of data from space science surveys. Generally speaking, any computational methodological tool performed to transform data into information is called a Data Mining System (DMS). It is notable that in space science (astronomy, astrophysics, cosmology and solar system studies) many existing data archives are unsuitable for DM because key pieces of metadata are missing. Hence, our goal in the first part of this project is to outline the major components of such a DMS, logically connected to the data processing and data grid requirements described previously.

\section{A NEW ERA FOR BRAVO}

In the past two years we have gained important experience and knowledge of $\mathrm{VO}$ development, and our project was realigned to be economically feasible. New collaborations were established in all facets of our planned investment. Within the context of the INCT-Astronomy, the priority is to devise a roadmap for the near future to coherently invest in hardware and software that can meet our researchers needs. BRAV0@INPE aims to create this synergy and contribute in strategic areas of the global VO.

Below we list the main strategic points of this enterprise. We emphasize that these are the overarching items defining this project and can be seen as pillars of a consistent investment in V0.

\subsection{Network Infrastructure}

From the results of our IT census we see the level of insufficiency of the hardware used by the astronomical community in Brazil, especially for astronomers located in smaller and more isolated institutes. High speed and secure network connections are of paramount importance not only for simple tasks in our daily work but also for establishing a national grid processing facility, such as the one we are developing with the BNPGA. The Brazilian National Research and Education Network (RNP) has been enhancing the network infrastructure throughout Brazil and has recently started the development of a plan to improve network access within the astronomical community in Brazil (see http://www.rnp.br/en/backbone/index.php). This is one 
of the main points of this project - to coordinate a study of the current situation and establish a schedule for implementing a modern network infrastructure for all institutes of astronomy in the country.

\subsection{Creating the BNPGA}

The census we did within the INCT-Astronomy community indicated that we need to both upgrade our network infrastructure and invest in creating a Grid Processing facility that can meet the growing demands of the astronomy community, not only because of the increase in the amount of data but also due to its increasing complexity. The BNPGA is the response to communities need for processing a large amount of data and reliably publishing the results in an environment meeting V0 standards. BNPGA will commence as an exercise of processing the entire SDSS in one band; by doing so we will be able to implement the environment before upgrading to more powerful clusters with thousands of modern processors.

\subsection{Astro-Wise as a national environment for data reduction and analysis}

Several pipelines were developed in recent years to address the demands of large area surveys like SDSS. In these cases, users do not have to worry about data reduction. However, more and more sophisticated algorithms for object detection, star-galaxy separation, photometric redshift estimates, morphological analysis and other tasks are flourishing and there is an obvious need for reprocessing data in some of these cases. As discussed above, we are implementing AW (developed to be VO compliant) as the environment for large amounts of data processing. Ours will be the first AW node in South America, as it is currently extant only in Europe in compliance with IVOA standards.

\subsection{The Virtual Lab for Advanced Data Analysis (VLADA)}

The Virtual Laboratory for Advanced Data Analysis is a project initiated at the Lab for Computing and Applied Mathematics-INPE which aims to provide a new virtual environment for scientific analysis tools to extract statistical and physical information from time series, images and hypercube data. Its preliminary version consists of a PHP user interface through which the user can input the data and receive specific measures characterizing the data (statistical moments, power spectra, generalized dimensions, EuIer characteristics, asymmetry coefficients, etc). In the context of BRAV0@INPE, VLADA can be seen as a virtual tool box for data analysis in general. VLADA will be made available as a VO service, which will require high speed connectivity and a high performance server. The project is being developed under the same umbrella as BRAV0@INPE since the technical operational issues are similar.

In order to tackle all of these issues within BRAV0@INPE we are planning to invest in personnel in three main categories. First, the project includes a collaboration with computer scientists from the Laboratory for Computing and Applied Mathematics at INPE (LAC) who are mainly engaged in projects dedicated to data mining and database development. They will be the main personnel involved in BRAV0@INPE making use of the large data sets acquired in the upcoming decades and pursuing solutions to problems astronomers will be facing in the Petabyte era. Second, LAC is recruiting staff specializing in database management, web design, and operating system management. They will be responsible for keeping the V0 services operational. Third, we will support graduate students and postdocs involved in the project. They will be developing their theses on subjects related to topics described in Section 7.

\section{SUMMARY}

The Virtual Observatory is rapidly becoming a reality. The combination of growing data volumes and data complexity, coupled with computational and algorithmic advances, has made the $\mathrm{VO}$ a necessity. We have described some of the ongoing projects to implement databases, general-purpose computational algorithms, grid networks, and other V0-enabling technologies in Brazil. A common theme among all of these developments is the dire need for computational resources (CPUs, storage and network), software, and the expertise to design, install, and bring to life these complex systems. The international nature of astronomy implies that everyone can benefit and everyone should contribute to this enterprise. We have described the specific contributions that the Brazilian astronomical and computer science communities have made and will be making to this effort. Our growing partnerships in large telescopes and unfettered access to large public data sets demands that we develop our own tools and expertise to leverage these investments and strengthen our scientific output. Finally, we have described the necessary next steps in terms of hardware, software and personnel to advance BRAV0@INPE from an incipient program to a fully functioning project.

\section{REFERENCES}

[1] BALL N et al. 2006. ApJ. 650, 497.

[2] BENACCHIO L \& PASIAN F. 2007. Grid-enabled Astrophysics, 
Contributions to the Computational Grids for Italian Astrophysics: Status and Perspectives workshop, held at INAF headquarters, Rome, in November 2005. Polimetrica.

[3] BISHOP CM. 1994. Rev. Sci. Instrum. 65, 1803.

[4] BISHOP CM. 2006. Pattern Recognition and Machine Learning. Springer

[5] CARETTA C et al. 2008. A\&A. 487, 445

[6] DJORGOVSKI SG et al. 1998. Wide Field Surveys in Cosmology, 89.

[7] DRYDEN IL. 2005. Annals of Statistics 33, 1643.

[8] FADDA D et al. 1996. ApJ, 473, 670

[9] FRANÇOIS D. 2008. "High-dimensional Data Analysis: From Optimal Metrics to Feature Selection". VDM Verlag.

[10] GARGIULO A et al. 2009. arXiv0902.4383.

[11] HANISCH R \& QUINN P. http://www.ivoa.net/pub/info/ThelVOA.pdf.

[12] HARTIGAN JA. 1975. Clustering Algorithms. New York, NY, USA: John Wiley \& Sons.

[13] HAYKIN S. 1994. Neural Networks: A Comprehensive Foundation, Macmillan, New York.

[14] HEGE HC \& POLTHIER K. 2002. Mathematical Visualization: Algorithms, Applications and Numerics. Springer.

[15] HERNANDEZ-TOLEDO HM. 2008. A\&A 136, 2115.

[16] HEY T, TANSLEY S \& TOLLE K. 2009. The Fourth Paradigm - DataIntensive Scientific Discovery, Microsoft Research.

[17] JAIN AK et al. 2000. IEEE Trans. on Pattern Analysis and Machine Intelligence, 22, 1.

[18] KATGERT P et al. 1996. A\&A, 310, 8.

[19] KUNTSCHE EN. 2003. Swiss Journal of Psychology/Schweizerische Zeitschrift für Psychologie/Revue Suisse de Psychologie, 62, 202.

[20] LA BARBERA F et al. 2008a. PASP, 120, 681.

[21] LA BARBERA F et al. 2008b. ApJL, 689, 913.

[22] LA BARBERA F et al. 2009. AJ 137, 3942.

[23] LA BARBERA F \& DE CARVALHO RR. 2009. ApJL, 699, 76.

[24] LOPES PAA et al. 2009. MNRAS, 392, 135.

[25] LOTZ JM et al. 2004. AJ, 128, 163.

[26] MERCURIO A et al. 2008. MNRAS, 387, 1374

[27] OLSEN LF et al. 2005. A\&A, 435, 781.

[28] QUINLAN JR. 1986. Machine Learning, 1, 81.
[29] QUINLAN JR. 1993. Programs for Machine Learning. San Mateo, CA: Morgan Kaufman.

[30] RIEBER LP. 1995. Educational Technology Research and Development, 43, 45.

[31] ROSA RR et al. 2007. Physica A 386, 666-673.

[32] RUIZ RSR et al. 2008. National Congress on Computing and Applied Mathematics,

www.sbmac.org.br/eventos/cnmac/xxxi_cnmac/PDF/251.pdf.

[33] SHEWCHUK JR. 1996. Applied Computational Geometry: Towards Geometric Engineering, Eds. Ming C. Lin and Dinesh Manocha, Vol 1148 of Lecture Notes in Computer Science, 203 Springer-Verlag.

[34] SOARES SANTOS M et al. 2008. arXiv:0810.3689.

[35] SUCHKOV AA. 2005. AJ, 130, 2439.

[36] TAFFONI G, BELIKOV A \& SCHAAFF A. 2009. Mem. S. A. It., 80, 493.

[37] TELEA AC. 2007. Data Visualization, AK Peters.

[38] VALENTIJN E et al. 2006. Astronomical Data Analysis Software and Systems XVI ASP Conference Series, Vol. 376, 2007, RA Shaw, F Hill and DJ Bell, eds.

[39] WOJTAK R et al. 2007. A\&A, 466, 437.

[40] WRIGHT H. 2006. Introduction to Scientific Visualization, Springer.

\section{APPENDIX}

\section{A. UNIVERSITIES AND INSTITUTES ASSOCIATED TO INCT-A}

\section{São Paulo}

USP - Universidade de São Paulo

INPE - Instituto Nacional de Pesquisas Espaciais

UPM - Universidade Presbiteriana Mackenzie

UNICSUL - Universidade Cruzeiro do Sul

UNIVAP - Universidade do Vale do Paraíba

UNESP - Universidade Estadual Júlio de Mesquita Filho

UNIFESP - Universidade Federal de São Paulo

UFABC - Universidade Federal do ABC

FSA - Fundação Santo André

\section{Rio Grande do Sul}

UFRGS - Universidade Federal do Rio Grande do Sul

UFSM - Universidade Federal de Santa Maria

UFPel - Universidade Federal de Pelotas

Unipampa - Universidade Federal do Pampa

UCS - Universidade de Caxias do Sul 


\section{Rio de Janeiro}

ON - Observatório Nacional

UFRJ - Universidade Federal do Rio de Janeiro

CBPF - Centro Brasileiro de Pesquisas Físicas

\section{Minas Gerais}

UFMG - Universidade Federal de Minas Gerais

LNA - Laboratório Nacional de Astrofísica

UNIFEI - Universidade Federal de Itajubá

UFJF - Universidade Federal de Juiz de Fora

\section{Santa Catarina}

UFSC - Universidade Federal de Santa Catarina

Unochapecó - Universidade Comunitária Regional de Chapecó

\section{Bahia}

UESC - Universidade de Santa Cruz

\section{Distrito Federal}

UNB - Universidade de Brasília

\section{Paraná}

UEL - Universidade Estadual de Londrina

\section{Pernambuco}

UNIVASF - Universidade Federal do Vale do São Francisco

\section{B. FIRST COMPUTING RESOURCES CENSUS OF THE INCT-A GROUPS}

We conducted a census with all 31 institutes associated to INCTAstronomy, asking specifically:

1) What is the total number of users they have in their Department?

2) How many computers do they have access to, including Desktops, and what are their main characteristics?

3) Do they have access to cluster systems? If so, what are the characteristics?

Although the questionnaire might not be very objective, the main idea was to collect as much info as possible and then try to organize it accordingly. Twenty (66\%) of the institutes participating of the INCT-Astronomy responded to the questions. The remaining institutes, which did not answer, represent small groups (2-3 researchers in located in Physics Departments) still involved in implementing basic infrastructure. Therefore, it would be fair to consider the data presented in the Tables 1-4 as representative of the cyber infrastructure of the Brazilian astronomical community.
Three types of information were requested and we present them in Tables 1-4: Number of users, including researchers and graduate students (Table 1); Cyber infrastructure available in terms of Desktops (Table 2); Cyber infrastructure available in terms of clusters (Tables 3a and 3b).

From Table 1 we see that $66 \%$ of the institutes composing the INCT-Astronomy contribute with 277 users. The remaining 12 institutes contribute with 30 users, making a total of 310 users who participate directly or indirectly of the INCT-Astronomy. The figure below shows the distribution of the number of users per institute from where we can see that although the Brazilian community had a very significant growth in the last 20 years, most of the main power working on astronomy in Brazil is still concentrated in a few places. This is something to be addressed in the near future within the context of a broad program being prepared by the INCT-Astronomy but it goes beyond the scope of this project.

From Table 2, we can conclude that most users of our community have access to at least a Desktop with moderate computational capacity. This conclusion must be seen with caution. It happens that the Brazilian astronomical community is dominated by stellar astrophysicists ( $70 \%$ ) doing important and competitive research but using data of low complexity (1D spectroscopy and/or 1D photometry etc). It is important to remember that even this situation is changing dramatically and will keep changing in the near future with the large telescopes coming online. In this context, we understand that the current computational facilities available seem to be adequate and fulfill the present demand. However, it is clear that the current Cyber infrastructure will be obsolete when dealing with the extremely large amount of data coming from either stellar or extragalactic projects.

Tables 3a and 3b refer to the info about clusters available, allowing high performance processing. As we can see, researchers from these 20 institutes have access to modern servers with more than 8 processors each (Class A) and to beowulf types, composed of mono-processed nodes and internal networks of $100 \mathrm{Mps}$ (Class B). Only 12 out of the 20 institutes listed in Tables $3 \mathrm{a}$ and $3 \mathrm{~b}$ have access to a cluster and only 7 out of these 12 have access to a Class A cluster. These numbers will not change considerably if we include the remaining 12 institutes which did not provide information.

In essence, $50 \%$ of the institutes composing the INCTAstronomy have access to a cluster, regardless of which class. It is important to note that in some cases the clusters are shared with researchers from different disciplines like Physics since the 
Table 1

\begin{tabular}{|c|c|c|c|c|}
\hline $\begin{array}{c}\text { INSTITUTE } \\
(1)\end{array}$ & $\begin{array}{l}\text { Users } \\
\text { (2) }\end{array}$ & $\begin{array}{c}\text { Researchers } \\
\text { (3) }\end{array}$ & $\begin{array}{c}\text { Visitors+ } \\
\text { Postdocs } \\
\text { (4) }\end{array}$ & $\begin{array}{c}\text { Students: } \\
\text { Grad+Msc+PhD } \\
\text { (5) }\end{array}$ \\
\hline USP/IAG & 67 & 22 & 10 & 35 \\
\hline USP/EACH & 1 & 1 & 0 & 0 \\
\hline LNA & [6] & [0] & $?$ & 0 \\
\hline USP/IF & 1 & 2 & $?$ & $?$ \\
\hline UFSC/DF & 23 & 4 & 1 & 18 \\
\hline UNIVAP/IP\&D & 13 & 6 & 0 & 7 \\
\hline INPE & 20 & 8 & 0 & 12 \\
\hline UFMG/DF & 22 & 8 & 2 & 12 \\
\hline UNICSUL/NucAstro & 7 & 7 & 0 & 0 \\
\hline UESC/DCET & 6 & 7 & 1 & 0 \\
\hline UFRGS/IF & 49 & 10 & 4 & 35 \\
\hline UFRJ/OV & 25 & 14 & 1 & 15 \\
\hline UNIMACK & 8 & 8 & $?$ & $?$ \\
\hline UFRJ/IF & 21 & 4 & 1 & 16 \\
\hline CAXIAS S. & 1 & 1 & 0 & 1 \\
\hline UNIFESP & 1 & 2 & $?$ & $?$ \\
\hline UFABC & 5 & 2 & $?$ & 3 \\
\hline UNIFEI & 4 & 4 & $?$ & $?$ \\
\hline UNIPAMPA & 3 & 3 & 0 & 0 \\
\hline ON & & $9 ?$ & $?$ & $?$ \\
\hline
\end{tabular}

Column (2) totalizes the content of the ensuing columns.

small groups of researchers developing Astronomy in Brazil are inserted in large Physics Departments.

Adding up all the available processors in the different clusters as listed in Tables $3 a$ and $3 b$, we would have in principle the total number of processors for grid processing (see Table 4). This total, 419 , is only $6 \%$ of the required number mentioned in the figures presented previously for processing the entire DR7 in one band, in one week, for example. This is only a crude estimate considering that all the processors are different, some better than others - fifty are old type of processors that would add little to the total processing capacity. In terms of total storage, these clusters do not go over 45TB, and although it satisfies the needs of individual groups, is clearly incompatible with the needs of the coming decade where large telescopes will produce data on a $2 \mathrm{~PB} /$ year rate.

Finally, we want to stress that this census although may not represent the entire Brazilian astronomical community, it shows how deficient the current hardware/software and network infrastructure is. 
Table 2

\begin{tabular}{|c|c|c|c|c|c|}
\hline INSTITUTE & Desktops & Processors & $\begin{array}{c}\text { Total RAM } \\
(\mathrm{Gb}) \\
(1)\end{array}$ & $\begin{array}{c}\text { Storage } \\
(\mathrm{Tb})\end{array}$ & OS \\
$(2)$ & $(3)$ & $(4)$ & $(6)$ \\
\hline USP/IAG & $?$ & 185 & {$[>185]$} & 29.6 & LWM \\
\hline USP/EACH & 2 & 2 & 3 & 3 & $?$ \\
\hline LNA & $?$ & $?$ & $?$ & $?$ & L[WM] \\
\hline USP/IF & $?$ & $?$ & $?$ & $?$ & L[WM] \\
\hline UFSC/DF & 18 & 35 & 16 & 2.5 & LW \\
\hline UNIVAP/IP\&D & 7 & 14 & $7 \times 1$ & $>7 \times 0.2$ & LW \\
\hline INPE & 21 & 30 & 30 & 4 & LW \\
\hline UFMG/DF & 12 & 17 & 25 & 2.6 & LW \\
\hline UNICSUL/NuCAstro & $6+1$ & $?$ & 18 & 2 & LW \\
\hline UESC/DCET & 21 & 28 & 20 & 1 & LW \\
\hline UFRGS/IF & $10-15$ & 22 & 13 & 1 & LW \\
\hline UFRJ/OV & 13 & 17 & $10+?$ & $0.8+?$ & LW \\
\hline UNIMACK & 12 & 12 & 18.5 & 9 & L \\
\hline UFRJ/IF & 21 & 21 & 30 & 1 & LW \\
\hline CAXIAS S. & $5+10$ & 8 & 5 & 1.5 & W \\
\hline UNIFESP & 3 & 3 & 6 & 0.75 & LW \\
\hline UFABC & 5 & $?$ & 10 & 6 & $?$ \\
\hline UNIFEI & 6 & 11 & 30 & 2 & LW \\
\hline UNIPAMPA & $?$ & $?$ & $?$ & $?$ & $?$ \\
\hline ON & 10 & 15 & 25 & 4 & LW \\
\hline
\end{tabular}

Items within brackets ([ ]) are our own estimates of not informed items. Column (6): $L=L$ inux; $\mathrm{W}=$ Ms-Windows; $\mathrm{M}=$ Mac OS. 
Table 3a

\begin{tabular}{|c|c|c|c|c|c|c|c|}
\hline $\begin{array}{c}\text { INSTITUTE } \\
\text { (1) }\end{array}$ & $\begin{array}{l}\text { CLUSTER } \\
\text { (2) }\end{array}$ & $\begin{array}{c}\text { Description } \\
\text { (3) }\end{array}$ & $\begin{array}{l}\text { Storage } \\
(\mathrm{Tb}) \\
(4)\end{array}$ & $\begin{array}{l}\text { Internal } \\
\text { Network } \\
\text { (5) }\end{array}$ & $\begin{array}{l}\text { OS } \\
\text { (6) }\end{array}$ & $\begin{array}{l}\text { Parallel } \\
\text { Software } \\
(7)\end{array}$ & $\begin{array}{c}\text { Installed } \\
\text { Software } \\
(8)\end{array}$ \\
\hline USP/IAG & $\begin{array}{c}\text { HYDRA } \\
\text { HPC } \\
\text { BEETHOVEN }\end{array}$ & $\begin{array}{c}20 \times 2 \text { Xeon/3Ghz } \\
\text { Itautec } \\
22 \times 1 \text { AMD64/3Ghz } \\
\text { 12×1 Intel/AMD } \\
3 \mathrm{Ghz}\end{array}$ & $\begin{array}{l}20 \times 0.2 \\
22 \times 0.16 \\
1 \times 0.12\end{array}$ & $\begin{array}{c}\text { Gigabit } \\
\text { [=Infiniband?] } \\
\text { Ethernet } \\
100 \mathrm{Mb} \\
\text { Ethernet } \\
100 \mathrm{Mb}\end{array}$ & $\begin{array}{c}\text { Ganglia } \\
+[\text { Linux }] \\
\text { Ganglia } \\
+[\text { Linux }] \\
\text { Ganglia } \\
+[\text { Linux }]\end{array}$ & $\mathrm{MPI}$ & \\
\hline USP/EACH & & & & & & & \\
\hline USP/IF & $\begin{array}{l}\text { PMC/ } \\
\text { DFMa }\end{array}$ & $\begin{array}{c}\text { 48×2/ 1Ghz } \\
\text { 1×48Gb/RAM } \\
+ \text { Server:10Gb/RAM }\end{array}$ & {$[<10]$} & $\begin{array}{l}\text { [Ethernet } \\
100 \mathrm{Mb} \text { ] }\end{array}$ & Linux & [VPM] & mathematica \\
\hline LNA & $\begin{array}{c}\text { HP } \\
\text { DL380R05 }\end{array}$ & $\begin{array}{c}1 \times \text { QuadCore } \\
\text { Xeon E5405/2.0Ghz }\end{array}$ & 12 & & [Linux] & & \\
\hline UFSC/DF & MINERVA & $\begin{array}{c}6 \times \text { HP(XeonE5405) } \\
+9 \times \text { AMD64/3Ghz } \\
+4 \times \text { Core2quad+storage }\end{array}$ & 3.5 & Gigabit[?] & $\begin{array}{l}\text { Ganglia } \\
+ \text { Cacti } \\
+ \text { pbs }\end{array}$ & & \\
\hline UNIVAP/IP\&D & [noname] & $7 \times$ Dual-Core/3Ghz & $7 \times 0.08$ & Trendnet & Linux & [MPI] & [gadget] \\
\hline INPE & CPAD/Inpe & $\begin{array}{c}23 \times 2+2 \times 4 \\
\text { Opteron } 2 \text { core } \\
+ \text { servidor +storage }\end{array}$ & 4.5 & $\begin{array}{l}\text { Infiniband } \\
\text { 2.5Gbps }\end{array}$ & $\begin{array}{l}\text { Linux } \\
\text { RedHat }\end{array}$ & MPI & $\begin{array}{l}\text { gadget } \\
\text { 2DPhot }\end{array}$ \\
\hline
\end{tabular}

Items within brackets ([ ]) are our own estimates of not informed items. / Column (2) gives the nicknames of the corresponding equipment; a bar (/) indicates that this is a shared resource. Notice that multi-processed $(\mathrm{N}>4)$ storage servers have also been included here. 
Table 3b

\begin{tabular}{|c|c|c|c|c|c|c|c|}
\hline $\begin{array}{c}\text { INSTITUTE } \\
\text { (1) }\end{array}$ & $\begin{array}{c}\text { CLUSTER } \\
\text { (2) }\end{array}$ & $\begin{array}{c}\text { Description } \\
\text { (3) }\end{array}$ & $\begin{array}{c}\text { Storage } \\
(\mathrm{Tb}) \\
(4)\end{array}$ & $\begin{array}{l}\text { Internal } \\
\text { Network } \\
(5)\end{array}$ & $\begin{array}{l}\text { OS } \\
\text { (6) }\end{array}$ & $\begin{array}{c}\text { Parallel } \\
\text { Software } \\
(7)\end{array}$ & $\begin{array}{c}\text { Installed } \\
\text { Software } \\
(8)\end{array}$ \\
\hline UFMG/DF & $\begin{array}{c}\text { \{BULL/ } \\
\text { University }\}\end{array}$ & $\begin{array}{c}\{50 \times 4 ? \\
; 50 \times 32 \mathrm{~Gb} / \mathrm{RAM}\}\end{array}$ & \{\} & \{Infiniband?\} & \{\} & & \\
\hline $\begin{array}{l}\text { UNICSUL/ } \\
\text { NucAstro }\end{array}$ & $\begin{array}{c}\text { estação1 } \\
\text { estação2 } \\
\{\}\end{array}$ & $\begin{array}{l}8 \times ? ; 16 \mathrm{~Gb} / \mathrm{RAM} \\
8 \times ? ; 32 \mathrm{~Gb} / \mathrm{RAM}\end{array}$ & $\begin{array}{l}1.5 \\
1.5\end{array}$ & & $\begin{array}{l}\text { Linux RedHat } \\
\text { Linux RedHat }\end{array}$ & & \\
\hline UESC/DCET & $\begin{array}{c}\text { [noname] } \\
\text { \{BULL/ } \\
\text { University\} }\end{array}$ & $\begin{array}{c}\text { 16×Athlon/1.7Ghz } \\
\text { /8GbRAM } \\
\{160 \times \text { Xeon/2.66Ghz } \\
\text { 320GbRAM+storage }\}\end{array}$ & $\{10\}$ & $\{$ infiniband $\}$ & $\{$ Linux; PBS-pro; Bull $\}$ & & $\{$ Oracle $\}$ \\
\hline UFRGS/IF & CPADA & $\begin{array}{c}6 \times 2 \times \text { Athlon } \\
\text { 1.4GHz/1GbRAM }\end{array}$ & 0.24 & $\begin{array}{l}\text { Ethernet } \\
100 \mathrm{Mb}\end{array}$ & Linux & MPI & Gadget \\
\hline UFRJ/OV & $\begin{array}{c}\text { [noname] } \\
\text { DELL }\end{array}$ & $\begin{array}{c}\text { 4×Celeron/2.4Ghz } \\
\text { /4×1GbRAM } \\
4 \times \text { Xeon; }\end{array}$ & $\begin{array}{l}0.32 \\
0.25\end{array}$ & $\begin{array}{l}\text { Ethernet } \\
100 \mathrm{Mb}\end{array}$ & $\begin{array}{c}\text { Rock's + Linux } \\
\text { Ubuntu }\end{array}$ & & \\
\hline UNIMACK & [noname] & $\begin{array}{l}\text { 15×2 Opteron/1.4Ghz } \\
\text { +server2core/?GbRAM }\end{array}$ & 1 & $?$ & Debian & & \\
\hline \multicolumn{8}{|l|}{ UFRJ/IF } \\
\hline CAXIAS & [noname] & $\begin{array}{c}11 \times \mathrm{P} 4 / 2.6 \mathrm{GHz} / \\
1 \mathrm{GbRAM}+1 \times \mathrm{P} 4 / 3 \mathrm{Ghz}\end{array}$ & $12 \times 0.08$ & $\begin{array}{l}\text { Ethernet } \\
100 \mathrm{Mb}\end{array}$ & Oscar-Linux & & \\
\hline UNIFESP & \{\} & & & & & & \\
\hline UFABC & \{\} & & & & & & \\
\hline \multicolumn{8}{|l|}{ UNIFEI } \\
\hline \multicolumn{8}{|l|}{ UNIPAMPA } \\
\hline ON & & & & & & & \\
\hline
\end{tabular}

Items within braces (\{ \}) correspond to projected acquisitions. / Items within brackets ([ ]) are our own estimates of not informed items. / Column (2) gives the nicknames of the corresponding equipment; a bar (/) indicates that this is a shared resource. Notice that multi-processed $(\mathrm{N}>4)$ storage servers have also been included here. 
Table 4

\begin{tabular}{|c|c|c|c|}
\hline INSTITUTE & Users & $\begin{array}{c}\text { No. Cluster } \\
\text { Processors }\end{array}$ & $\begin{array}{c}\text { Cluster } \\
\text { Storage (Tb) }\end{array}$ \\
\hline USP/IAG & 67 & 74 & 7.64 \\
\hline USP/EACH & 1 & 0 & 0 \\
\hline LNA & 6 & 4 & 12 \\
\hline USP/IF & 1 & 96 & 10 \\
\hline UFSC/DF & 23 & 31 & 3.5 \\
\hline UNIVAP/IP\&D & 13 & 14 & 0.56 \\
\hline INPE & 20 & 108 & 4.5 \\
\hline UFMG/DF & 22 & 0 & 0 \\
\hline UNICSUL/NuCAstro & 7 & 16 & 3 \\
\hline UESC/DCET & 6 & 16 & 1 \\
\hline UFRGS/IF & 49 & 12 & 0.24 \\
\hline UFRJ/OV & 25 & 8 & 0.57 \\
\hline UNIMACK & 8 & 32 & 1 \\
\hline UFRJ/IF & 21 & 0 & 0 \\
\hline CAXIAS S. & 1 & 12 & 0.96 \\
\hline UNIFESP & 1 & 0 & 0 \\
\hline UFABC & 5 & 0 & 0 \\
\hline UNIFEI & 4 & 0 & 0 \\
\hline UNIPAMPA & 3 & 0 & 0 \\
\hline ON & {$[9]$} & 0 & 0 \\
\hline Totals & 283 & 423 & 44.97 \\
\hline UIII & 0 & & 0 \\
\hline
\end{tabular}

Items within brackets ([ ]) are our own estimates of not informed items. 